\title{
Zeit für ein erstes Resümee
}

Anfang 2007 haben Sie das erste Heft der neu konzipierten Flugmedizin Tropenmedizin Reisemedizin (FTR) erhalten. Ziel war es damals gewesen, den wissenschaftlichen Teil des Heftes stärker in den Mittelpunkt zu stellen. Die Vertreter der verschiedenen Fachgesellschaften im Editorial Board haben auf einer Sitzung jetzt im April 2008 festgestellt, dass die Zeitschrift wirklich attraktiv geworden ist - und wir hoffen alle, dass Sie als Leser das genauso sehen. An dieser Stelle möchte ich Frau Kleinlein vom Georg Thieme Verlag danken, die sich unermüdlich um die Qualität der Zeitschrift bemüht.

Aber natürlich wollen wir die Qualität der Flugmedizin Tropenmedizin Reisemedizin weiter verbessern. Wir planen, zwei neue Rubriken einzuführen, die Rubrik „Journal-Club“ und die Rubrik „Kasuistik“. Im Journal-Club stellen die einzelnen Fachrichtungen eine aktuelle, möglicherweise bahnbrechende Arbeit vor, vielleicht auch einen Artikel, der für besonders kontroverse Diskussionen gesorgt hat. In der Rubrik „Kasuistik“ können interessante Fälle dargestellt werden.

Wenn Sie als Leser hier Vorschläge haben, lassen Sie es uns bitte wissen. Die Beiträge aus den beiden Rubriken werden genauso den Reviewprozess durchlaufen wie die anderen Beiträge. Des Weiteren werden wir von Zeit zu Zeit ein Schwerpunktheft konzipieren, in dem ein Thema von den verschiedenen Fachgesellschaften bearbeitet werden soll - für die Ausgabe 4/2008 haben wir das Thema Diabetes mellitus vorgesehen.

An dieser Stelle möchte ich Sie auch noch einmal darauf hinweisen, dass die FTR online zugänglich ist. Die Startseite der Zeitschrift lautet www.thieme.de/flugundreise. Über den Button „Inhaltsverzeichnis“ erreicht man die aktuelle Ausgabe der FTR. Gesellschaftsmitglieder und Abonnenten können sich den kompletten Inhalt der Zeitschrift mithilfe ihrer Abonummer kostenfrei ansehen und die einzelnen Beiträge als PDF herunterladen.

Ich hoffe zusammen mit den anderen Mitgliedern des Editorial Board, dass Ihnen die aktuelle Ausgabe der FTR gefällt und freue mich darauf, die Zeitschrift mit Ihrer Unterstützung weiter zu entwickeln.
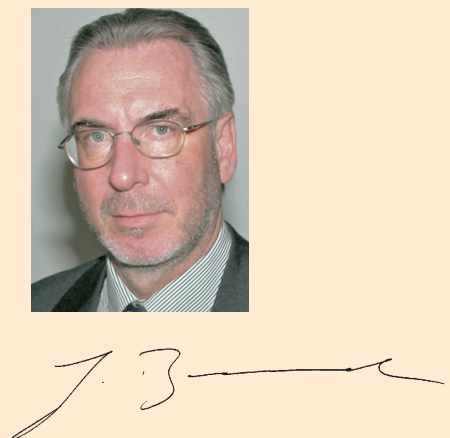

Prof. Dr. Gerd-Dieter Burchard, Hamburg 